\title{
Kopf-Hals-Tumoren: Ergebnisse zum Langzeitüberleben veröffentlicht
}

\begin{abstract}
- Die American Society of Clinical Oncology (ASCO) hat Cetuximab (Erbitux ${ }^{\circledR}$ ) wiederholt als einen der bedeutenden Fortschritte in der Krebstherapie eingestuft. Dieses Jahr wurde Cetuximab von der ASCO ausgewählt, da dies die erste Therapieoption seit 30 Jahren ist, die in der Erstlinienbehandlung von Patienten mit rezidivierten und/oder metastasierten Plattenepithelkarzinomen des Kopfes und Halses (SCCHN) einen signifikanten Überlebensvorteil zeigen konnte. Der ASCO-Bericht „Clinical Cancer Advances 2009: Major Research Advances in Cancer Treatment, Prevention and Screening" ist eine unabhängige Bewertung
\end{abstract}

der wichtigsten klinischen Studien in der Krebsforschung des vergangenen Jahres. Zeitgleich mit der Einstufung wurden auch die Fünf-Jahres-Überlebensdaten der randomisierten Phase-III-Studie von Bonner et al., welche die Wirksamkeit von Cetuximab beim lokal fortgeschrittenen SCCHN untersuchte, in The Lancet Oncology publiziert. Die aktuelle Langzeitanalyse bestätigt den Einsatz von Cetuximab in Kombination mit Strahlentherapie in der Behandlung des lokal fortgeschrittenen SCCHN: So lebten fast die Hälfte der Patienten, die Cetuximab in Kombination mit Strahlentherapie erhielten, nach fünf Jahren noch - im Vergleich zu einem Drittel der Patienten, die mit alleiniger Strahlentherapie behandelt wurden ( $45,6 \%$ vs. $36,4 \%$; $p=0,018$ ). Die Hinzunahme von Cetuximab zur Strahlentherapie führte zu einem anhaltenden Überlebensvorteil (OS 49,0 Monate vs. 29,3 Monate; HR 0,725; $p=0,018)$. Zudem korreliert das Auftreten von akneiformen Hautreaktionen mit einem zusätzlichen Überlebensvorteil, wodurch das Mortalitätsrisiko um 51\% reduziert werden konnte.

Nach Informationen von Merck Serono, Darmstadt

\section{Hereditäres Angioödem wird oft fehlgedeutet}

— Das Hereditäre Angioödem (HAE) wird aufgrund seiner Seltenheit (ca.1.50o Betroffene in Deutschland) häufig verkannt. Oft werden die Hautschwellungen zunächst als allergisches Quincke-Ödem fehlgedeutet. Die Erkrankung wird in der Regel autosomal vererbt, kann aber auch aufgrund einer Neumutation ausbrechen. Sie beruht auf einem Mangel oder einer funktionellen Störung des $\mathrm{C}_{1}$-Esterase-Inhibitors ( $\left.\mathrm{C}_{1}-\mathrm{INH}\right)$. Typische Symptome sind häufig wiederkehrende Schwellungen an Händen, Füßen oder im Gesicht wie auch an Schleimhäuten, berichtete Dr. Emel Aygören-Pürsün,
Frankfurt/Main. Sind die Schleimhäute im Bereich des Kehlkopfs betroffen, kann eine Attacke sogar lebensbedrohlich sein, eine Schwellung der Darmschleimhaut kann zum Darmverschluss führen. Häufig kommt es auch zu krampfartigen Bauchattacken. Die Ödeme bilden sich in der Regel innerhalb von einem bis acht Tagen zurück, kommen jedoch häufig wieder. Bei Verdacht auf HAE muss die Diagnose per Bluttest gesichert werden.

An der HAE-Ambulanz in Frankfurt, der weltweit größten seiner Art, werden inzwischen mehr als 500 HAE-Patienten behandelt.
Therapie der Wahl ist hier die i.v.-Injektion eines C1-INH-Eiweißkonzentrats (Berinert ${ }^{\circledR}$ P). Die Substitutionstherapie sollte individuell erfolgen, da die Attackenfrequenz stark variiert, riet Dr. Inmaculada Martinez-Saguer.Spüren die Patienten erste Symptome, sollten sie sich das Präparat injizieren. Die Therapie sei gut verträglich.

Pressegespräch „Schnelle Hilfe bei schwerer, seltener Erkrankung“, Frankfurt am Main, 21. Januar 2010 Veranstalter: CSL Behring, Marburg

\section{Bessere Emesis-Prophylaxe bei Hochdosis-Chemotherapie}

— Für Patienten mit hämatologischen Neoplasien, die vor hämatopoetischer Stammzelltransplantation (HSCT) hoch emetogene Hochdosis-Chemotherapien (HC) zur myeloablativen Konditionierung erhalten, sind Übelkeit und Erbrechen ein großes Problem, das mit 5-HT3-Antagonist und Dexamethason nicht ausreichend kontrolliert werden kann. Der Neurokinin-1-Rezeptorantagonist Aprepitant $\left(\right.$ Emend $\left.^{\circledR}\right)$ hat sich bereits bei hoch emetogenen auf Cisplatin basierenden und moderat emetogenen Chemotherapien in konventioneller Dosierung zur Prävention der zytostatika-induzierten Emesis bewährt und wurde daher nun in einer Phase-III-Studie auch im Rahmen verschiedener hoch emetogener HC mit oder ohne Ganzkörperbestrahlung vor HSCT geprüft. In die monozentrische Doppelblindstudie wurden 179 Patienten mit unterschiedlichen hämatologischen Neoplasien eingeschlossen, die randomisiert entweder das Dreierregime mit Aprepitant (125 mg am Tag 1, danach 80 mg täglich während und 3 Tage nach $\mathrm{HC}$ ), Ondansetron und Dexamethason (beides täglich während und 1 Tag nach $\mathrm{HC}$ ) oder Ondansetron, Dexamethason plus Placebo erhielten. Den primären Endpunkt (= kein Erbrechen und keine oder nur leichte Übelkeit) erreich- ten im Aprepitant-Arm mit 82\% signifikant mehr Patienten als im Kontrollarm (66\%; $p<$ $0,001) .49 \%$ der mit dem Aprepitant-Regime, aber nur $15 \%$ der mit dem Zweierregime behandelten Patienten litten im Studienverlauf weder unter Erbrechen noch unter Übelkeit. Besonders ausgeprägt war der Effekt von Aprepitant auf die Emesis: Bei 73\% der Patienten im Aprepitant-Arm im Vergleich zu $23 \%$ im Kontrollarm trat im Studienverlauf überhaupt kein Erbrechen auf.

Nach Informationen von MSD Sharp \& Dohme, Haar 